\title{
Microbiology of urine samples obtained through suprapubic bladder aspiration: A 10-year epidemiological snapshot
}

\author{
M GAJDÁCS ${ }^{1 *}, \mathrm{M}^{\mathrm{A} B R O ́ K}{ }^{2}, \mathrm{~A} \mathrm{LÁZÁR}^{2}$ and $\mathrm{K}$ BURIÁN ${ }^{2,3}$ \\ ${ }^{1}$ Department of Pharmacodynamics and Biopharmacy, Faculty of Pharmacy, University of Szeged, Szeged, Hungary \\ ${ }^{2}$ Faculty of Medicine, Institute of Clinical Microbiology, University of Szeged, Szeged, Hungary \\ ${ }^{3}$ Department of Medical Microbiology, Faculty of Medicine, University of Szeged, Szeged, Hungary
}

(Received: September 3, 2019; revised manuscript received: October 9, 2019; accepted: November 16, 2019)

\begin{abstract}
Purpose: Suprapubic bladder aspiration is an invasive procedure in which a needle is used to obtain a urine sample directly from the bladder. Its advantages are sensitivity (detection of significant bacteriuria is close to $100 \%$ ), suitability for cultivation of anaerobic bacteria, and low risk of contamination. Our purpose was to characterize the microbiology and epidemiology of urine samples obtained through this procedure in the Clinical Center of the University of Szeged's Institute of Clinical Microbiology between 2008 and 2017. Materials and methods: Over the 10-year period, patient data were collected and suprapubic bladder aspirations were performed, and the samples are processed in accordance with routine laboratory procedures in clinical bacteriology. Results: Of 187 urine samples obtained from 148 patients, $32.6 \%(n=61)$ were culture-positive (defined as $10^{2}$ colony forming units $/ \mathrm{ml}$ or more). Conclusions: This method should be considered an important sampling procedure in the differential diagnostics of upper urinary tract infections, particularly in children $<2$ years of age, and in older people, hospitalized patients.
\end{abstract}

Keywords: suprapubic bladder aspiration, urinary tract infections, upper UTIs, epidemiology, matrix-assisted laser desorption/ionization-time-of-flight

\section{INTRODUCTION}

Suprapubic bladder aspiration (i.e., a bladder tap, as first described by Guze \& Beeson [1]) is an invasive procedure in which a needle is used to obtain a urine sample directly from the bladder [2]. The advantages of this method include its sensitivity (detection of significant bacteriuria is close to $100 \%$ ), suitability for cultivation of anaerobic bacteria, and low risk of contamination $[2,3]$. In this study, we present the culture results and related epidemiology of urine samples obtained through this procedure in a teaching hospital in Hungary from 2008 to 2017.

\section{MATERIALS AND METHODS}

This study was carried out with the cooperation of the University of Szeged's Institute of Clinical Microbiology and the Albert Szent-Györgyi Clinical Center, a 1,820-bed teaching hospital providing primary and tertiary care for the urban and rural population in Csongrád County, Hungary. We performed a retrospective microbiological characterization of urine samples obtained through suprapubic bladder aspiration at the Clinical Center over a 10-year period (between January 1, 2008 and December 31, 2017). Patient data were collected, limited to demographic characteristics (age and gender) and the indication for sample submission [4].
Urine samples were processed in accordance with routine procedures in clinical bacteriology [5]. Facultative anaerobes were cultivated using UriSelect chromogenic agar plates (Bio-Rad, Berkeley, CA, USA) at $37{ }^{\circ} \mathrm{C}$ in a $5 \%$ $\mathrm{CO}_{2}$ atmosphere. As per request of the clinicians, some of the urine samples were also cultivated anaerobically, with the help of an anaerobic chamber (Baker Ruskinn, York, UK), in an atmosphere of $90 \% \mathrm{~N}_{2}, 5 \% \mathrm{H}_{2}$, and $5 \% \mathrm{CO}_{2}$ at $37{ }^{\circ} \mathrm{C}$. Between 2008 and 2012, bacterial isolates were identified based on presumptive (biochemical) methods and VITEK 2 Compact ID (facultative anaerobes) and ANC (strict anaerobes) cards (bioMérieux Hungária Ltd., Budapest, Hungary). From 2012 onward, they were identified using matrix-assisted laser desorption/ionization-timeof-flight mass spectrometry, using the Microflex MALDI Biotyper (Bruker Daltonics, Bremen, Germany). The methodology of sample preparation for mass spectrometry measurements was described elsewhere [6]. Facultative anaerobes were tested for antibiotic susceptibility using the disk diffusion method on Mueller-Hinton agar plates, whereas anaerobic isolates were tested using E-test (Liofilchem, Abruzzo, Italy) on Brucella blood agar (BBA, Oxoid,

* Corresponding author: Márió Gajdács, PharmD, PhD; Department of Pharmacodynamics and Biopharmacy, Faculty of Pharmacy, University of Szeged, Eötvös utca 6, Szeged H-6720, Hungary; E-mail: mariopharma92@gmail.com

This is an open-access article distributed under the terms of the Creative Commons Attribution 4.0 International License, which permits unrestricted use, distribution, and reproduction in any medium, provided the original author and source are credited, a link to the CC License is provided, and changes - if any - are indicated. (SID_1) 
Basingstoke, UK) supplemented with 5\% laked sheep blood, hemin, and vitamin $\mathrm{K}$ [7]. Interpretation of the susceptibility results and detection of resistance mechanisms were based on EUCAST guidelines (http://www.eucast.org/). Descriptive statistical analysis (including means or medians with ranges and percentages to characterize data) was performed using Microsoft Excel 2013 (Microsoft Corp., Redmond, WA, USA).

\section{RESULTS}

A total of 187 urine samples $(18.7 \pm 8.4$ year; range: $4-29$; lowest in 2014, highest in 2017), obtained through suprapubic bladder aspiration from 148 patients, were received during the study period (2008-2017). During that 10-year period, the Institute received 19,325 samples from inpatient departments and 21,150 samples from outpatient clinics of the Albert Szent-Györgyi Clinical Center; therefore, $0.5 \%$ of urine samples were obtained through suprapubic bladder aspiration [8]. The patients had an average age of 33.4 years (median: 11; range: $0-84$ ) and a male-to-female ratio of 1.88 $(65.2 \%$ male). Most affected patients were younger than $5(41.7 \%)$ years or older than $50(39.4 \%)$ years. Most of the samples $(95.9 \%)$ were originated from the Departments of Pediatrics (54.6\%), Internal Medicine (20.7\%), Urology $(15.5 \%)$, and the Intensive Care Unit (5.1\%). The indications for sample submission (based on the submission forms provided with the samples) were mainly related to disorders of the urinary system $(87.7 \%)$; these included congenital abnormalities, suspicions of urinary tract infections (UTIs), kidney stones, or malignancy and renal failure. The other indications (12.3\%) were traumatic injuries, septicaemia, and stroke.

Overall, $32.6 \%$ of the samples $(n=61)$ were culturepositive (defined as $10^{2}$ colony forming units $/ \mathrm{ml}$ or more), which is in line with data in the literature for culture positivity of urine samples $(10 \%-50 \%)$ and the results in our Institute for conventionally collected urine samples ( $\sim 34 \%-38 \%$; for these samples, culture positivity is usually defined as $10^{5}$ or more colony forming units/ml) $[8,9]$. In the positive urine samples, the following species were isolated in the order of decreasing frequency: Enterococcus faecalis $(n=14)$, Escherichia coli $(n=10)$, Klebsiella pneumoniae $(n=8)$, Pseudomonas aeruginosa $(n=8)$, Proteus vulgaris $(n=6)$, Enterococcus faecium and Staphylococcus aureus ( $n=3$ each), Enterobacter cloacae, Candida albicans, Candida tropicalis, and Proteus mirabilis ( $n=2$ each), and Klebsiella oxytoca $(n=1)$. In addition, $28.9 \%(n=54)$ of the samples were cultivated anaerobically, per request of the treating physician, and three more species were isolated, such as Finegoldia magna $(n=2)$, Peptococcus niger, and Peptinophilus indolicus ( $n=1$ each). None of the positive samples included more than one isolate. Multidrug-resistant (MDR) pathogens were recovered from 11 samples (18.0\%), namely, the extended-spectrum $\beta$-lactamase producing $E$. coli $(n=5), K$. pneumoniae $(n=3)$, and E. cloacae $(n=1)$, the vancomycin-resistant $E$. faecium $(n=1)$, and the methicillin-resistant $S$. aureus $(n=1)$. The susceptibilities of the remaining pathogens were unremarkable.

\section{DISCUSSION}

In this study, we characterized the microbiology and epidemiology of urine samples obtained between 2008 and 2017 through suprapubic aspiration of the bladder of patients in the southern region of Hungary. This was the first such study in Hungary and the longest-spanning study of its kind overall. The main indications for the suprapubic aspiration method included urinary retention (e.g., due to hyperplasia or tumours of the prostate, gynaecological malignancies, or spinal cord injuries) and constriction, phimosis, and chronic (therapyresistant) infections or congenital abnormalities of the urinary system in young children and older adults [10]. In addition, this sampling method may be used in the diagnosis of lower UTIs, where obtaining a sample voluntarily or through catheterization is not possible $[2,10]$. With the appropriate equipment (disinfectant, local anaesthetic, 22-gauge spinal needle, syringe, and sterile collection container) and clinical experience, obtaining the sample is a relatively simple procedure and provides clinically relevant information [11]. Nevertheless, as it is an invasive sampling procedure, some disadvantages (fear of the patient, pain, and discomfort) and possible complications (haematuria, bleeding, visceral injuries, and hemoperitoneum in severe cases) should be considered [12].

Interestingly, one out of six isolates in this study was MDR. This may present serious therapeutic challenges for clinicians, as they will not only have to consider the pharmacokinetics of the drugs appropriate for these infections, but they will also have a limited drug armamentarium due to the resistance $[13,14]$. There is no known pathological mechanism or epidemiological evidence in the literature to explain the accumulation of MDR strains in these infections. During the 10 -year study period, several anaerobic species were detected from bladder tap samples, presumably corresponding to chronic upper UTIs. The latter finding is especially interesting, as there is mounting evidence that anaerobes may be significant pathogens in UTIs; however, because urine samples are not routinely cultured in an anaerobic environment, more studies (especially employing molecular biological methods or sequencing) are needed to ascertain the real clinical role of these microorganisms [2, 3, 9]. Among other anaerobes, Actinotignum schaalii (formerly known as Actinobaculum, first described in 1997) has received substantial attention as an important urinary tract pathogen in elderly patients or in people with underlying urological pathologies [15]. This Gram-positive bacterium (as are other anaerobes in the urinary tract) is often overlooked. Relevant urine samples are usually present with a negative nitrite test and significant leukocyturia; molecular methods may also be used, but for verification of infection, cultivation of the sample in strict anaerobic conditions is a compulsory [16].

\section{CONCLUSIONS}

Suprapubic bladder aspiration should be considered as an important sampling procedure in the differential diagnostics of upper UTIs, particularly in children $<2$ years of age (where conventional sampling methods may prove 
ineffective), in older people, hospitalized patients, or if chronic infections caused by anaerobes are suspected.

Acknowledgements: Part of this study was presented at the 46th Congress of the Hungarian Society of Infectious Disease Physicians and Clinical Microbiologists (MIFKMT; Velence, Hungary).

Authors' contributions: MG conceived and designed the study. MÁ and AL were the senior microbiologists and performed the identification and of the bacterial isolates and interpreted susceptibility-testing results during the study period. MG, MÁ, and AL performed data collection and analysis, and involved in writing and revision of the full paper. $\mathrm{KB}$ wrote and revised the manuscript.

Ethical approval: The study was deemed exempt from ethics review by the Institutional Review Board, and informed consent was not required as data anonymity was maintained.

Conflicts of Interest/Funding: The authors declare no conflict of interest, monetary, or otherwise. MG was supported by the National Youth Excellence Scholarship (grant number: NTP-NTFÖ-18-C-0225) and the ESCMID Mentorship and Observership Programme.

\section{REFERENCES}

1. Guze LB, Beeson PB. Observations on the reliability and safety of bladder catheterization for bacteriologic study of the urine. N Eng J Med. 1956;255(10):474-5.

2. Ponka D, Baddar F. Suprapubic bladder aspiration. Can Fam Physician. 2013;59(1):50.

3. Rozenfeld K, Nitzan O, Peretz A. Presence of anaerobic bacteria in the urinary tract of catheterized ICU patients. Eur J Clin Microbiol Infect Dis. 2018;37(11):2131-6.
4. Gajdács M. Resistance trends and epidemiology of Aeromonas and Plesiomonas infections (RETEPAPI): a 10-year retrospective survey. Infect Dis (London). 2019;51(9):710-3.

5. Gajdács M, Dóczi I, Ábrók M, Lázár A, Burián K. Epidemiology of candiduria and Candida urinary tract infections in inpatients and outpatients: results from a 10-year retrospective survey. Cent Eur J Urol. 2019;72(2):209-14.

6. Gajdács M, Spengler G, Urbán E. Identification and antimicrobial susceptibility testing of anaerobic bacteria: Rubik's cube of clinical microbiology? Antibiotics (Basel). 2017;6(4):E25.

7. Gajdács M, Urbán E. The relevance of anaerobic bacteria in brain abscesses: a ten-year retrospective analysis (2008-2017). Infect Dis (London). 2019;51(10):779-81.

8. Gajdács M, Ábrók M, Lázár A, Burián K. Resistance trends and epidemiology of Citrobacter-Enterobacter-Serratia in urinary tract infections of inpatients and outpatients (RECESUTI): a 10-year survey. Medicina (Kaunas). 2019;55(6):E285.

9. Flores-Mireles AL, Walker JN, Caparon M, Hultgren SJ. Urinary tract infections: epidemiology, mechanisms of infection and treatment options. Nat Rev Microbiol. 2015;13(5):269-84.

10. Eliacik K, Kanik A, Yavascan O, et al. A comparison of bladder catheterization and suprapubic aspiration methods for urine sample collection from infants with a suspected urinary tract infection. Clin Pediatr (Phila). 2015;55(9):819-24.

11. Marin JR, Shaikh N, Docimo SG, et al. Suprapubic bladder aspiration. N Engl J Med. 2014;371:E13.

12. Pollack CV Jr, Pollack ES, Andrew ME. Suprapubic bladder aspiration versus urethral catheterization in ill infants: success, efficiency, and complication rates. Ann Emerg Med. 1994;23(2):225-30.

13. Abbo LM, Hooton TM. Antimicrobial stewardship and urinary tract infections. Antibiotics (Basel). 2014;3:174-92.

14. Gajdács $M$. The concept of an ideal antibiotic: implications for drug design. Molecules. 2019;24(5):892.

15. Cattoir V. Actinobaculum schaalii: review of an emerging uropathogen. J Infect. 2012;64(3):260-7.

16. Prigent G, Perillaud C, Amara M, Coutard A, Blanc C, Pangon B. Actinobaculum schaalii: a truly emerging pathogen? New Microbes New Infect. 2016;11:8-16. 